\title{
A via das quinureninas e suas implicações na esquizofrenia
}

\author{
Kynurenine pathway and its implications in schizophrenia \\ Samuel Frota Cunha ${ }^{1}$, Tatiana Paschoalette Rodrigues Bachur², Gislei Frota Aragão $0^{2 *}$ \\ ${ }^{1}$ Graduado em Medicina; ${ }^{2}$ Doutora em Farmacologia
}

\begin{abstract}
Resumo
Introdução: a esquizofrenia é uma doença mental grave heterogênea, de extrema complexidade, manifestada por vários distúrbios de cognição, pensamento, memória, comportamento e afeto. Sua fisiopatologia, apesar dos avanços, continua de difícil compreensão. Objetivo: realizar uma revisão da literatura a respeito da relação entre a via das quinureninas e a fisiopatologia da esquizofrenia. Metodologia: foi conduzida a busca de artigos através das bases de dados Medline e Lilacs, utilizando os descritores schizophrenia/ esquizofrenia e as palavras-chave kynurenine pathways/via das quinureninas. Por meio desta busca, foram selecionados 38 artigos publicados entre os anos de 1990 e 2018. Resultados: estudos mais recentes vêm demonstrando que anormalidades no metabolismo do triptofano por meio da via das quinureninas poderiam estar relacionadas com os mecanismos neurofisiopatológicos da esquizofrenia. Os metabólitos gerados pela via das quinureninas possuem propriedades neuroativas. Dentre eles, destaca-se o ácido quinurênico, antagonista endógeno do receptor N-metil-D-aspartato. Seus níveis cerebrais estão aumentados em pacientes esquizofrênicos, provavelmente devido a uma condição pró-inflamatória, resultando dessa forma, em possíveis alterações neurológicas responsáveis pelo desenvolvimento da esquizofrenia. Conclusão: não somente uma melhor compreensão da fisiopatologia da esquizofrenia, como também o desenvolvimento de novos alvos terapêuticos, poderão ser obtidos a partir de um melhor entendimento da relação entre anormalidades na via das quinureninas e a gênese da esquizofrenia.

Palavras-chave: Esquizofrenia. Triptofano. Metabolismo.
\end{abstract}

\begin{abstract}
Introduction: Schizophrenia is a severe heterogeneous mental illness of extreme complexity, manifested by various disorders of cognition, thought, memory, behavior and affection. Its pathophysiology, despite advances, remains difficult to understand. Objective: to review the literature on the relationship between the kynurenine pathway and the pathophysiology of schizophrenia. Methodology: a literature review was carried out using the descriptors kynurenine pathways/via das quinureninas and the keywords kynurenine pathways/via das quinureninas using Medline and Lilacs databases. Through this search, were selected 38 articles published between the years of 1990 and 2018. Results: recent studies have shown that abnormalities in tryptophan metabolism through the kynurenine pathway may be related to the neuropathophysiological mechanisms of schizophrenia. The metabolites generated by the kynurenine pathway have neuroactive properties. Among them, kynurenic acid, an endogenous antagonist of the N-methyl-D-aspartate receptor, stands out. Their brain levels are increased in schizophrenic patients, probably due to a pro-inflammatory condition, resulting in possible neurological changes responsible for the development of schizophrenia. Conclusion: not only a better knowledge of pathophysiology, but also the development of new therapeutic targets will be obtained with a better understanding of the relationship between abnormalities in the kynurenine pathway and the genesis of schizophrenia.

Keywords: Schizophrenia. Tryptophan. Metabolism.
\end{abstract}

\section{INTRODUÇÃO}

A esquizofrenia é uma doença mental crônica de extrema complexidade e gravidade, manifestada por distúrbios cognitivos, de memória, pensamento e volição. $O$ termo esquizofrenia foi introduzido por Eugen Bleuler (1857 - 1939) para definir a expressão "quando alguém não pode dizer a diferença entre o que é real e imaginário", ou seja, significa a presença de cisões (schisms) entre pensamento, emoção e comportamento (FREEDMAN, 2003; SADOCK, B.; SADOCK. V.; RUIZ, 2017).

A essência da esquizofrenia pode ser resumida como um processamento anormal de informações no cérebro.

Correspondente/Corresponding: *Gislei Frota Aragão - Universidade Estadual do Ceará - CCS - Coordenação de Medicina - End: Av. Dr. Silas Munguba, 1700, Itaperi CEP 60.914-703 - Tel : (85) 3101-9732 - E-mail: gislei.frota@uece.br
Entretanto, longe dessa simplicidade, sua fisiopatologia ainda permanece nebulosa e como um grande desafio para a ciência. Isso decorre do fato de que uma infinidade de síndromes discretamente distintas entre si, com mecanismos fisiopatológicos diferentes são simplesmente sumarizadas em uma única entidade: a esquizofrenia. Acredita-se, pelo menos, que sua gênese decorra da interação entre determinados fatores biológicos, ambientais e genéticos (KAHN et al., 2015; MULLER; MYINT; SCHWARZ, 2011)

No final do século passado, olhares se voltaram para a via das quinureninas na fisiopatologia de doenças psiquiátricas e neurológicas (MULLER; MYINT; SCHWARZ, 2011). Essa via é a responsável pela degradação do aminoácido triptofano, tendo como principal produto a coenzima nicotinamida adenina dinucleotídeo $-\mathrm{NAD}^{+}$. No entanto, 
além deste, são gerados metabólitos com propriedades neuroativas que podem modular o estresse oxidativo e a neurotransmissão, podendo estarem relacionados com os mecanismos neurobiológicos resultantes da esquizofrenia (CHIAPPELLI et al., 2018; SCHWARCZ et al., 2012)

Diante disso, a melhor compreensão da via das quinureninas, especialmente seus metabólitos, e suas relações com os mecanismos neurofisiopatológicos da esquizofrenia abre campo para a exploração de novos alvos terapêuticos passíveis de serem utilizados nesse transtorno.

\section{METODOLOGIA}

O presente artigo constituiu em uma revisão narrativa de literatura realizada através da busca de artigos dentro da temática de estudo. Foram conduzidas buscas nas bases de dados Medline e Lilacs, utilizando como descritores schizophrenia/esquizofrenia, e as palavras-chave kynurenine pathway/via das quinureninas. Os critérios de inclusão para a seleção foram: artigos originais e de revisão escritos nos idiomas inglês e português, com ano de publicação entre 1990 e 2018 e com temática referente ao assunto estudado. Foram excluídos manuscritos em outros idiomas e com ano de publicação fora do período estabelecido (a não ser que de grande relevância para o tema). Outrossim, foram utilizados livros textos, bem consolidados na psiquiatria, para construção desse artigo.

A partir da pesquisa, obtiveram-se 38 artigos então utilizados para produção desta revisão.

\section{RESULTADOS E DISCUSSÃO}

\section{Esquizofrenia}

A esquizofrenia é uma doença igualmente prevalente entre homens e mulheres. Difere, porém, entre os sexos, quanto a idade de início dos sintomas. Homens possuem incidência mais precoce, entre os 10 e 25 anos; mulheres, por outro lado, iniciam a doença entre os 25 e 35 anos. O início dos sintomas antes dos 10 ou após os 60 anos é raro (SADOCK, B.; SADOCK. V.; RUIZ, 2017).

A qualidade de vida é gravemente afetada. Cerca de $80 \%$ das pessoas acometidas apresentam dificuldade para retornar às atividades realizadas prévias à doença. Outrossim, a redução na expectativa de vida em aproximadamente 20 anos é outro problema importante (HARROW et al., 1997; LAURSEN; NORDENTOFT; MORTENSEN, 2014) Isso se deve não só a um aumento nas taxas de suicídio, como também a um incremento na morte por causas naturais, devido, por exemplo, a doença cardiovascular prematura induzida por tratamento com antipsicóticos, adoção de hábitos de vida não saudáveis e menor acesso a serviços de saúde (AMERICAN PSYCHIATRY ASSOCIATION, 2013; BROWN; INSKIP; BARRACLOUGH, 2000; MCCREADIE, 2003)

O diagnóstico é complexo e essencialmente clínico, baseado na história psiquiátrica e no exame do estado mental. O uso de exames de imagem, laboratoriais e genéticos pode auxiliar de alguma forma no raciocínio clínico, no entanto não estabelecem diagnóstico (SADOCK, B.; SADOCK. V.; RUIZ, 2017).

As diferenças são claras quanto a arquitetura celular, a conectividade da substância branca e o volume da substância cinzenta entre grupos de indivíduos saudáveis e aqueles com esquizofrenia. Observa-se redução volumétrica dos lobos temporais, frontais e parietais, bem como uma aceleração do processo de perda do parênquima (AMERICAN PSYCHIATRY ASSOCIATION, 2013; STEEN et al., 2006). Além dessa alteração estrutural, estudos de tomografia por emissão de pósitrons (PET) demonstraram aumento dos níveis sinápticos de dopamina no núcleo estriado ventral e redução de seus níveis no córtex frontal (KEGELES et al., 2010). Em contrapartida, estudos com espectroscopia em ressonância magnética evidenciaram aumento nos níveis de glutamato nas regiões pré-frontais e temporais mediais (POELS et al., 2013). Todos esses achados em exames de imagem são capazes de diferenciar pacientes com transtornos psicóticos daqueles não afetados, embora infelizmente não sejam sensíveis ou específicos o suficiente para estabelecerem qualquer diagnóstico (LIEBERMAN; FIRST, 2018).

A esquizofrenia é categoricamente composta por sintomas positivos, negativos e cognitivos. Sintomas positivos são constituídos por alterações no comportamento e pensamento, tais como a psicose, as alucinações e o discurso desorganizado. Por outro lado, os sintomas negativos constituem a chamada síndrome amotivacional, caracterizada por isolamento social, anedonia, embotamento afetivo e avolição. Por fim, os sintomas cognitivos são expressos como um conjunto amplo de disfunções cognitivas (KAHN et al., 2015).

Uma disfunção na via dopaminérgica daria gênese aos sintomas positivos. Contudo, somente esta alteração não elucida a presença nem dos sintomas negativos, nem dos cognitivos (PLITMAN et al., 2017). O que dá suporte a esse fato, é justamente a melhora parcial, ou mesmo a ausência de melhora do quadro clínico em pacientes tratados somente com antipsicóticos antidopaminérgicos (SUZUKI et al., 2012).

Sob outra perspectiva, a esquizofrenia também poderia ser resultante de uma desregulação do sistema glutamatérgico, tendo, pois, uma explicação mais abrangente quanto à sintomatologia do paciente. Uma hipofunção nos receptores $\mathrm{N}$-metil-D-aspartato levaria ao surgimento de todos os sintomas: positivos, negativos e cognitivos (BUBENÍKOVÁ-VALEŁOVÁ et al., 2008).

$O$ tratamento deve ser feito de maneira individualizada, avaliando-se as características clínicas e a rede de suporte disponível. O objetivo final é a recuperação funcional e social do indivíduo. Os tratamentos imediatos variam de acordo com a fase: aguda (pacientes em crise) ou de manutenção (pacientes remitidos). De uma maneira geral, o uso de antipsicóticos é obrigatório nesses dois momentos. O maior número de recaídas está associado 
a pior evolução, maior taxa de resistência ao tratamento e a uma redução do volume em regiões corticais, reforçando a importância do controle dos sintomas ao longo da evolução da doença (ANDREASEN et al., 2013; VAN HAREN et al., 2007).

Tanto antipsicóticos de primeira geração (típicos), quanto os de segunda geração (atípicos) podem ser utilizados para tratamento. São similares na eficácia, mas apresentam diferentes efeitos colaterais e, consequentemente, diferenças quanto à tolerabilidade. Os antipsicóticos de primeira geração, a exemplo do haloperidol e da clorpromazina, frequentemente induzem ao aumento da prolactina e produzem sintomas extrapiramidais (SEP). Os de segunda geração, tais como a risperidona e o aripiprazol, apresentam menor potencial para induzir SEP, no entanto estão associados ao maior ganho de peso e ao risco de síndrome metabólica (BERBERIAN et al., 2009; BITTER et al., 2013).

Apesar da evolução no tratamento farmacológico no último século, o desfecho funcional da esquizofrenia parece não ter melhorado. Pacientes que sofrem deste mal ainda esperam melhores alternativas para tratamento e cura desta doença, o que reforça a necessidade de novos estudos para tentar responder as várias lacunas que ainda existem em relação à fisiopatologia da esquizofrenia e seu tratamento.

\section{Via das quinureninas}

A maior parte do triptofano é metabolizado por meio da via das quinureninas. Como resultado, ocorre a formação de metabólitos neuroativos potencialmente relacionados com a disfunção cognitiva de transtornos mentais, como a esquizofrenia (SCHWARCZ et al., 2012).

A via das quinureninas (Figura 1) é iniciada com a oxidação do triptofano a $\mathrm{N}$-formilquinurenina por 3 enzimas: indoleamina 2,3-dioxigenase 1 (IDO1), indoleamina 2,3-dioxigenase 2 (IDO2) ou triptofano 2,3-dioxigenase (TDO2). Em seguida, uma desformilação da $\mathrm{N}$-formilquinurenina, por meio da formamidase, produz a quinurenina. Esta, por sua vez, é passível de ser metabolizada pelas 4-quinurenina-aminotransferases (KATs), pela quinurenina 3-monooxigenase (KMO), ou pela quinureninase. Cada uma gera respectivamente: o ácido quinurênico (KYNA), a 3-hidroxiquinurenina (3-HK) e o ácido antranílico. A 3-HK sofre ação da quinureninase, levando a formação do ácido 3-hidroxiantranílico, que, finalmente é convertido a ácido quinolínico (QUIN) (DOUNAY; TUTTLE; VERHOEST, 2015; PLITMAN et al., 2017).
Figura 1 - Via das quinureninas. IDO1 - indoleamina 2,3-dioxigenase 1. IDO2 - indoleamina 2,3-dioxigenase 2. TDO - triptofano 2,3-dioxigenase. KMO - quinureninas 3-monooxigenase. KAT-4-quinurenina-aminotransferase

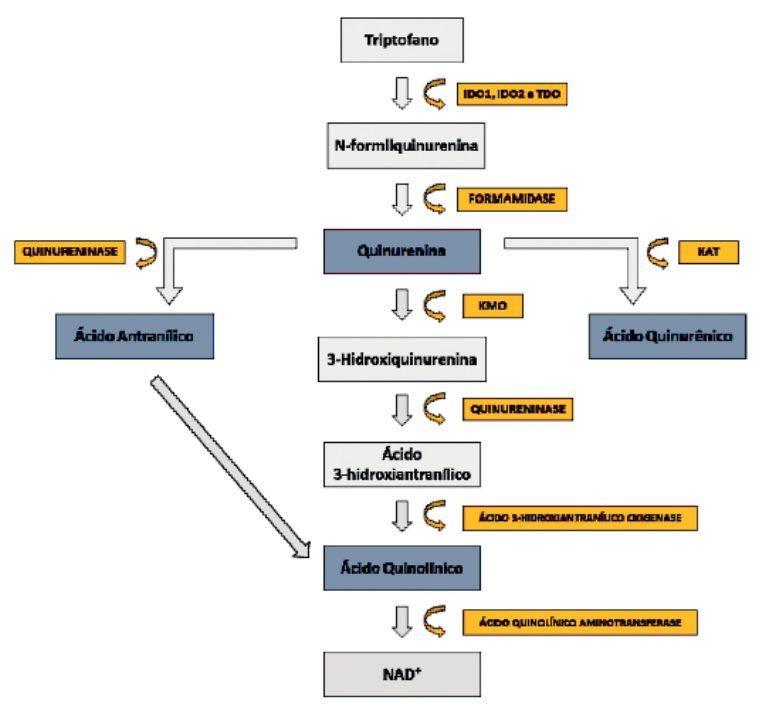

Fonte: Elaborada pelos autores.

Apesar da metabolização do triptofano à quinurenina ocorrer principalmente fora do sistema nervoso central (SNC), esta substância consegue atravessar com facilidade a barreira hematoencefálica. Em contrapartida, o ácido quinurênico, pela sua natureza polar, não a transpassa, tendo origem, portanto, na degradação da quinurenina já presente no SNC em meio ao ambiente astrocitário (GUILLEMIN et al., 2001; SCHWARCZ et al., 2012). O ácido quinolínico, por sua vez, é sintetizado na micróglia e em outras células de origem monocítica (HEYES et al., 1996).

Estudos sugerem que pacientes com esquizofrenia possuem níveis neurais aumentados de ácido quinurênico, o único antagonista endógeno conhecido dos receptores $\mathrm{N}$-metil-D-aspartato (Figura 2) (LINDERHOLM et al., 2010). Esse metabólito também atua como antagonista em mais dois outros receptores inotrópicos do glutamato (receptor do ácido $\alpha$-amino-3-hidroxi-5-metil-4-isoxazolepropionico e receptores cainatos) e do receptor $\alpha 7$ nicotínico $(\alpha 7 n A C h R)$. Age ainda como agonista do receptor associado a proteína G GPR35 - presentes em células do sistema imunológico - e do receptor aril hidrocarboneto (HILMAS et al., 2001; SUZUKI et al., 2012; WANG et al., 2006). Outrossim, além da capacidade de interação com tais receptores, o ácido quinurênico possui propriedades antioxidantes (LUGO-HUITRÓN et al., 2011). 
Figura 2 - Ação dos metabólitos da via das quinureninas nos mecanismos de neurotransmissão. $O$ ácido quinurênico atua como antagonista dos receptores NMDAr e $\alpha 7$ nicotínicos. Por outro lado, o ácido quinolínico atua como agonista dos receptores NMDAr. NMDAr - receptor N-metil-D-aspartato; $\alpha 7 n A C h R$ - receptor $\alpha 7$ nicotínico

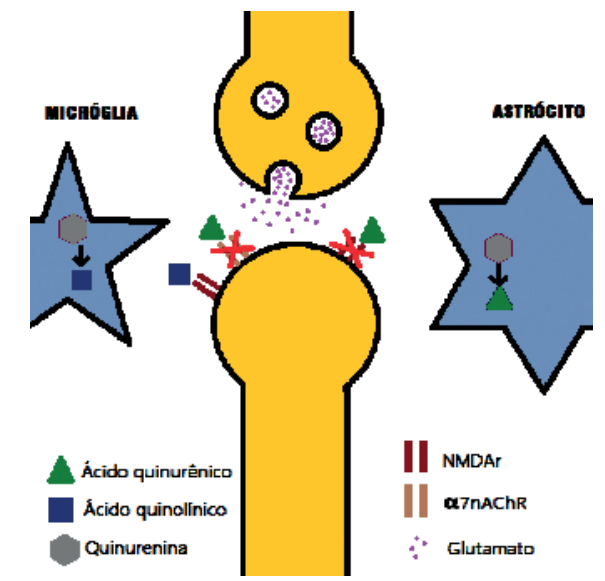

Fonte: Elaborada pelos autores.

Um aumento nos níveis de interleucina 6 (IL-6), interferon gama (IFN- $\gamma$ ) e fator de necrose tumoral alfa (TNF- $\alpha$ ), resultantes de uma atividade pró-inflamatória encontrada em indivíduos esquizofrênicos, causaria um incremento nos níveis cerebrais de KYNA, devido a uma maior ativação da enzima IDO. Consequentemente, pela propriedade antagonista do ácido quinurênico, haveria uma diminuição da atividade dos receptores NMDA, promovendo não só alterações na conectividade neural que culminariam com o desenvolvimento da esquizofrenia, como também uma exacerbação da doença pré-existente (CHIAPPELLI et al., 2018; FUJIGAKI et al., 2006; PLITMAN et al., 2017).

Com estudos recentes questionando a suposta exacerbação na atividade periférica da IDO e tal elevação dos níveis de IL-6 (CHIAPPELLI et al., 2018), uma alternativa para a explicação do aumento na concentração de ácido quinurênico poderia estar relacionado com uma redução na expressão e atividade cerebral do gene KMO, uma vez que com a ausência da enzima resultante, haveria direcionamento do metabolismo da quinurenina para a formação de KYNA (LAVEBRATT et al., 2013).

$O$ consequente hipofuncionamento dos receptores NMDA em interneurônios GABAérgicos inibitórios resultaria no aumento dos níveis pré-sinápticos de glutamato em várias regiões cerebrais, e a consequente manifestação de psicose e alterações corticolímbicas neurodegenerativas (OLNEY, 1995)

Estudos experimentais em ratos evidenciaram que um aumento nos níveis cerebrais de KYNA, principalmente durante a adolescência, levaram não somente a prejuízos cognitivos, como também na memória de trabalho, interação social e no circuito de recompensa. Esses prejuízos são semelhantes aos que ocorrem em pessoas com esquizofrenia (CHESS et al., 2007; TRECARTIN; BUCCI, 2011; TUFVESSON-ALM et al., 2018)
O aumento nos níveis endógenos de ácido quinurênico produz, também, uma significante ação nos neurônios dopaminérgicos, resultando em hiperatividade mesocorticolímbica, o que representa uma condição fisiopatológica semelhante àquela vista em pacientes esquizofrênicos (ERHARDT; ENGBERG, 2002; ERHARDT et al., 2007).

Os demais metabólitos gerados pela via das quinureninas, como a 3-hidroxiquinurenina (3-HK), o ácido 3-hidroxiantranílico (3-HANA) e o ácido quinolínico (QUIN) são todos neurotóxicos. O ácido quinolínico ativa seletivamente os receptores NMDA, além de causar rápida degeneração corticoestriatal em ratos, quando em níveis levemente elevados. Também estimula a liberação sináptica de glutamato e inibe a recaptação astroglial desse neurotransmissor (MADDISON; GIORGINI, 2015; TAVARES et al., 2002).

A regressão do estresse oxidativo, das mudanças comportamentais, do prejuízo cognitivo e uma diminuição da atividade motora foi possível com a utilização de inibidores das enzimas TDO, IDO e KMO em modelos animais de esquizofrenia induzidos com cetamina. Evidenciou-se, dessa forma, a possibilidade do surgimento de novos alvos terapêuticos para o tratamento desse transtorno mental (RÉUS et al., 2018).

\section{CONCLUSÃO}

Cada vez mais percebemos avanços científicos que provam uma relação direta entre alterações da via das quinureninas com a fisiopatologia da esquizofrenia. $\mathrm{O}$ melhor entendimento dessa via e da relação de seus metabólitos com o meio neural é essencial para a busca de novos alvos terapêuticos capazes de melhorar a morbimortalidade associada a esquizofrenia. Estudos devem ser direcionados principalmente para o entendimento das ações do sistema imunológico sobre os metabólitos da via das quinureninas, e quais são as alterações genéticas que estariam relacionadas com uma anormalidade no seu funcionamento.

\section{REFERÊNCIAS}

ANDREASEN, N. C. et al. Relapse duration, treatment intensity, and brain tissue loss in schizophrenia: a prospective longitudinal MRI study. Am. J. Psychiatr., Arlington, v. 170, n. 6, p.609-615, June 2013.

AMERICAN PSYCHIATRY ASSOCIATION. Diagnostic and statistical manual of mental disorders. 5. ed. Washington DC: American Psychiatry Association, 2013.

BERBERIAN, A. A. et al. Working memory assessment in schizophrenia and its correlation with executive functions ability. Rev. Bras. Psiquiatr., São Paulo, v. 31, n. 3, p.219-226, Set. 2009.

BITTER, I. et al. Comparative effectiveness of depot and oral second generation antipsychotic drugs in schizophrenia: a nationwide study in Hungary. Eur. Neuropsychopharmacol., Amsterdam, v. 23, n. 11, p.1383-1390, nov. 2013.

BROWN, S.; INSKIP, H.; BARRACLOUGH, B. Causes of the excess mortality of schizophrenia. Br. J. Psychiatr., London, v. 177, n. 3, p.212-217, Sept. 2000 . 
BUBENÍKOVÁ-VALEŁOVÁ, V. et al. Models of schizophrenia in humans and animals based on inhibition of NMDA receptors. Neurosci. Biobehav. Rev., New York, v. 32, n. 5, p.1014-1023, July 2008.

CHESS, A. C. et al. Elevations of Endogenous Kynurenic Acid Produce Spatial Working Memory Deficits. Schizophr. Bull., Rockville, v. 33, n. 3, p.797-804, 19 mar. 2007.

CHIAPPELLI, J. et al. Influence of plasma cytokines on kynurenine and kynurenic acid in schizophrenia. Neuropsychopharmacol., New York, v. 43, n. 8, p.1675-1680, 27 Feb. 2018.

DOUNAY, A. B.; TUTTLE, J. B.; VERHOEST, P. R. Challenges and Opportunities in the Discovery of New Therapeutics Targeting the Kynurenine Pathway. J. Med. Chem., Washingtom, v. 58, n. 22, p.8762-8782, 5 Ago. 2015.

ERHARDT, S.; ENGBERG, G. Increased phasic activity of dopaminergic neurones in the rat ventral tegmental area following pharmacologically elevated levels of endogenous kynurenic acid. Acta Physiol. Scand., Stockholm, v. 175, n. 1, p.45-53, May 2002.

ERHARDT, S. et al. The kynurenic acid hypothesis of schizophrenia. Physiol. Behav., Elmsford, v. 92, n. 1-2, p.203-209, Sept. 2007.

FREEDMAN, R. Schizophrenia. N. Engl. j. med., Boston, v. 349, n. 18, p.1738-1749, 30 out. 2003.

FUJIGAKI, H. et al. the signal transducer and activator of transcription $1 \alpha$ and interferon regulatory factor 1 are not essential for the induction of indoleamine 2,3-dioxygenase by lipopolysaccharide: involvement of p38 mitogen-activated protein kinase and nuclear factor-kb pathways, and synergistic effect of several proinflammatory cytokines. J. Biochem., Tokyo, v. 139, n. 4, p.655-662, 1 Apr. 2006.

GUILLEMIN, G. J. et al. Kynurenine pathway metabolism in human astrocytes: a paradox for neuronal protection. J. Neurochem., Oxford, v. 78, n. 4, p.842-853, 20 dez. 2001.

HARROW, M. et al. Course and outcome for schizophrenia versus other psychotic patients: a longitudinal study. Schizophr. Bull., Rockville, v. 23, n. 2, p.287-303, 1 jan. 1997.

HEYES, M. P. et al. Human microglia convert l-tryptophan into the neurotoxin quinolinic acid. J. Biochem., Tokyo, v. 320, n. 2, p.595-597, 1 Dec. 1996.

HILMAS, C. et al. The Brain Metabolite Kynurenic Acid Inhibits $\alpha 7$ Nicotinic Receptor Activity and Increases Non- $\alpha 7$ Nicotinic Receptor Expression: Physiopathological Implications. J. Neurosc., Baltimore, v. 21, n. 19, p.7463-7473, Oct. 2001.

KAHN, R. S. et al. Schizophrenia. Nat. Rev. Dis. Primers, [s.I.], v. 1, n. 1, p.1-23, 12 Nov. 2015.

KEGELES, L. S. et al. Increased Synaptic Dopamine Function in Associative Regions of the Striatum in Schizophrenia. Arch. Gen. Psychiatr., Chicago, v. 67, n. 3, p.231-239, 1 Mar. 2010.

LAURSEN, T. M.; NORDENTOFT, M.; MORTENSEN, P. B. Excess early mortality in schizophrenia. Ann. Rev. Clin. Psychol., Palo Alto, v. 10, n. 1, p.425-448, 28 Mar. 2014.

LAVEBRATT, C. et al. The KMO allele encoding Arg452 is associated with psychotic features in bipolar disorder type 1 , and with increased CSF KYNA level and reduced KMO expression. Mol. Psychiatr., Houndmills, v. 19, n. 3, p.334-341, 5 Mar. 2013.

LIEBERMAN, J. A.; FIRST, M. B. Psychotic disorders. N. Engl. J. Med.,Boston, v. 379, n. 3, p.270-280, 19 July 2018.

LINDERHOLM, K. R. et al. Increased levels of kynurenine and kynurenic acid in the csf of patients with schizophrenia. Schizophr. Bull., Rockville, v. 38, n. 3, p.426-432, 20 Ago. 2010.
LUGO-HUITRÓN, R. et al. On the antioxidant properties of kynurenic acid: Free radical scavenging activity and inhibition of oxidative stress. Neurotoxicol. Teratol., New York, v. 33, n. 5, p.538-547, Sep. 2011.

MADDISON, D. C.; GIORGINI, F. The kynurenine pathway and neurodegenerative disease. Semin. Cell Dev. Biol., London, v. 40, p.134141, Apr. 2015.

MCCREADIE, R. G. Diet, smoking and cardiovascular risk in people with schizophrenia. Br. J. Psychiatr., London, v. 183, n. 6, p.534-539, Dez. 2003.

MULLER, N.; MYINT, Aye-mu; SCHWARZ, M. J. Kynurenine Pathway in Schizophrenia: Pathophysiological and Therapeutic Aspects. Curr. Pharm. Des., Schiphol, v. 17, n. 2, p.130-136, 1 Jan. 2011.

OLNEY, J. W. Glutamate receptor dysfunction and schizophrenia. Arch. Gen. Psychiatr., Chicago, v. 52, n. 12, p.998-1007, 1 Dec. 1995.

PLITMAN, E. et al. Kynurenic acid in schizophrenia: a systematic review and meta-analysis. Schizophr. Bull., Rockville, v. 43, n. 4, p.764-777, 10 Feb. 2017.

POELS, M. P. et al. Imaging glutamate in schizophrenia: review of findings and implications for drug discovery. Mol. Psychatry., Houndmills, v. 19, n. 1, p.20-29, 29 Oct. 2013.

RÉUS, G. Z. et al. The inhibition of the kynurenine pathway prevents behavioral disturbances and oxidative stress in the brain of adult rats subjected to an animal model of schizophrenia. Progr. Neuropsychopharmacol. Biol. Psychiatry, Oxford, v. 81, p.55-63, Feb. 2018.

SADOCK, B.; SADOCK, V.; RUIZ, P. Transtornos do espectro da esquizofrenia e outros transtornos psicóticos. In: Compêndio de psiquiatria: ciência do comportamento e psiquiatria clínica. 11. ed. Porto Alegre: Artmed, 2017.

SCHWARCZ, R. et al. Kynurenines in the mammalian brain: when physiology meets pathology. Nat. Rev. Neurosc., London, v. 13, n. 7, p.465-477, 8 June 2012.

STEEN, R. G. et al. Brain volume in first-episode schizophrenia. Br. J. Psychiatr., London, v. 188, n. 6, p.510-518, June 2006.

SUZUKI, T. et al. Defining treatment-resistant schizophrenia and response to antipsychotics: A review and recommendation. Psychiatry Res., Amsterdam, v. 197, n. 1-2, p.1-6, May 2012.

TAVARES, R. G. et al. Quinolinic acid stimulates synaptosomal glutamate release and inhibits glutamate uptake into astrocytes. Neurochem. Int., Oxford, v. 40, n. 7, p.621-627, June 2002.

TRECARTIN, K. V.; BUCCI, D. J. Administration of kynurenine during adolescence, but not during adulthood, impairs social behavior in rats. Schizophr. Res., Amsterdam, v. 133, n. 1-3, p.156-158, Dez. 2011.

TUFVESSON-ALM, M. et al. Importance of kynurenine 3-monooxygenase for spontaneous firing and pharmacological responses of midbrain dopamine neurons: Relevance for schizophrenia. Neuropharmacology, Oxford, v. 138, p.130-139, Ago. 2018.

VAN HAREN, N. e M. et al. Focal gray matter changes in schizophrenia across the course of the illness: a 5-year follow-up study. Neuropsychopharmacol., New York, v. 32, n. 10, p.2057-2066, 28 Feb. 2007.

WANG, J. et al. Kynurenic acid as a ligand for orphan g protein-coupled receptor GPR35. J. Biol. Chem., Baltimore, v. 281, n. 31, p.22021-22028, 5 June 2006.

Submetido em: 16/09/2019

Aceito em: 06/02/2020 